\title{
Curve-fitting of Cam Profile and its Improvement
}

\author{
Dongfang Hu, Jianwei Guo
}

School of Mechatronics Engineering, Henan University of Science and Technology, Luoyang, Henan, 471003

\begin{abstract}
Based on the cubic spline interpolation for curve-fitting of molded lines of cam, an optimization method to reduce deviation of cam profile curve-fitting is raised. Calculate the given data of lift table by means of cubic spline interpolation and use curvature comb function of software UG to have a fairness analysis for cam profile curve. Cam profile curve will be produced after a calculation of cam lift data by cubic spline function. Then, utilize the curvature comb function of software UG to analyze the deviation of curve-fitting of the profile curve and implement a faring processing. With a contrast and analysis of curvatures before and after the faring processing, it is clear that cam profile curve has been improved obviously, which proves the method to be effective.
\end{abstract}

Keywords: Cam; Curve-Fitting of molded lines; Cubic spline; Deviation; Fairness

\section{凸轮轮廓的曲线拟合及其优化}

\author{
胡东方，郭建伟 \\ (河南科技大学机电工程学院, 河南 洛阳 471003)
}

摘要：基于凸轮型线拟合的三次样条插值方法, 提出凸轮轮廓曲线拟合误差的优化方法, 利用三次样条插值法对已知 升程表数据进行插值计算, 并用 UG 软件的曲率梳功能对凸轮的轮廓曲线进行光顺分析, 通过三次样条函数对凸轮升程数据 表进行插值拟合, 得到凸轮的轮廓曲线, 再根据 UG 曲率梳功能对轮廓曲线进行曲线拟合误差分析和光顺处理, 通过对光顺 前后曲率的比对分析，发现凸轮轮廊曲线得到了明显改善，进而验证了该方法的有效性。

关键词: 凸轮; 型线拟合; 三次样条; 误差; 光顺

中图分类号：TH 132.47 文献标志码：A

引言

凸轮轴是典型的异形回转零件，其轮廓具有比较复杂的几何曲线，而且凸轮型线对配气机构 ${ }^{[1]}$ 的工作 性能有着决定性影响。从加工的角度来说, 凸轮轮廓曲线光滑程度不仅影响着它的加工工艺, 对切削速度、 加工编程、刀具路径及优化手段等也有重要的影响 ${ }^{[2]}$, 所以凸轮轮廓曲线设计是凸轮轴生产过程中的关键 步骤。其中凸轮型线设计主要是设计其轮廓曲线, 其表示方法分为函数表示法 ${ }^{\left[{ }^{[3]}\right.}$ 和离散点升程表示法 ${ }^{[4]}$ 。 本文主要进行的是对于离散点升程表表示的凸轮数控加工的研究, 对于列表曲线加工的最大特点就是: 在 保证一定加工精度的前提下, 要求曲线能平滑地通过各个坐标点。并且对于列表曲线的数学处理, 通常采 用二次拟合法。

\section{1 凸轮轮廓曲线拟合}

对于列表曲线的数学处理, 通常采用二次拟合法 ${ }^{[5]}$ 。首先选择多个插值方程来分段描述它, 称为第一 次拟合，采用直线、圆弧插补方法来逼近插值方程所描述的曲线称为第二次拟合。为了保证第一次拟合的 
平滑性、连续性, 第一次拟合的算法显得特别重要列表曲线的方法很多, 但是应用于数控系统插补的较为 广泛的方法是三次样条插值 ${ }^{[6]}$ 和 $N$ 次谐波拟合方法 ${ }^{[7]}$ 。

\section{1 三次样条插值拟合}

三次样条拟合曲线 ${ }^{[8]}$ 能通过所有拟合点, 和原曲线有相同的凸凹性, 驻点数目相等, 且位置尽可能与 原曲线相同，拟合函数一致逼近原曲线，各型值点的一、二阶导数连续，极值性、收玫性和逼近性良好。 对于给定的凸轮轮廓曲线, 用三次样条拟合的方法, 可实现多段曲线间的平滑过渡连接, 以提高凸轮轮廓 精度。三次样条函数利用给定的离散型值点来进行插值计算。它是目前数控加工过程中, 解決列表曲线拟 合与插值问题时最被推崇的一种方法。三次样条插值函数的求解通常分为以下三个步骤:

第一步, 建立基本的方程组。插值函数 $S^{\prime}(x)$ 在小区间 $\left[x_{i-1}, x_{i}\right](i=1,2,3, \cdots \cdots, n)$ 上是三次多 项式, 该区间上其二阶导数值 $S^{\prime}(\mathrm{x})$ 是 $x$ 的一次多项式 (线性函数)。设 $S^{\prime}(x)$ 在 $x_{i-1}$ 处的函数值为 $M_{i}$, 则 由拉格朗日 (Lagrange) 线性插值定理可得:

$$
\left.S^{\prime \prime}(x)=M_{i} \frac{x_{i+1-x}}{h i}+M_{i+1} \frac{x-x i}{h i} \quad \text { (其中 } \mathrm{h}_{i}=\mathrm{x}_{i-1}\right)
$$

将上式积分两次后，把插值条件 $S\left(\mathrm{x}_{i-1}\right)=\mathrm{y}_{i-1}, S\left(x_{i}\right)=y_{i}$ 代入就可以得到 $S(x)$ 的“M 表达式”:

$$
S(x)=M_{i-1} \frac{\left(x_{i}-x\right)^{3}}{6 h_{i}}+M_{i} \frac{x-x_{i-1}}{h i}+\left(y_{i-1}-\frac{M_{i-1}}{6} h_{i}^{2}\right) \frac{x_{i}-x}{h i}+\left(y_{i}-\frac{M_{i}}{6} h_{i}^{2}\right) \frac{x-x_{i-1}}{h i}
$$

式中 $x \in\left[x_{i-1}, x_{i}\right]$ 。 $(i=1,2,3, \cdots, n)$

由以上可知, 公式中既保证了所求函数在各小区间上是一个三次多项式的插值函数, 还保证了函数在 样条节点处的连续性, 这样就保证了函数整个区间上的连续性。由于不知道 $S(x)$ 在节点处的二阶导数, 需 要求出这些待定系数。求这些二阶导数的待定系数时, 可用 $S(x)$ 在节点处的连续性特性, 来确定这些参数 所满足的关系式。对上式求导得:

$$
S^{\prime}(x)=-M_{i-1} \frac{\left(x_{i}-x\right)^{2}}{h_{i}}+M_{i} \frac{\left(x-x_{i-1}\right)^{2}}{2 h_{i}}+\frac{y_{i}-y_{i-1}}{h_{i}}-\frac{M_{i}-M_{i-1}}{6} h_{i}
$$

式中, $x \in\left[x_{i-1}, x_{i}\right]$ 。 $(\mathrm{i}=1,2,3, \cdots, n)$, 经左右求导最终可得三次样条插值函数的基本方程组为:

$$
\mu_{i} M_{i-1}+2 M_{i}+\lambda_{i} M_{i+1}=d_{i} \quad(i=1,2, \cdots, n-1)
$$

式中, $d_{i=6[}\left[\frac{y_{i+1}-y_{i}}{h_{i+1}}-\frac{y_{i}-y_{i-1}}{h_{i}}\right] / h_{i}+h_{i+1}=6 f\left[x_{i-1}, x i, x_{i+1}\right]$ 。

第二步, 建立端点条件。对于基本方程组的方程个数比待求参数少, 无法解出方程的情况, 就要结合 具体问题增加两约束条件或者减少两待定参数来求解方程组。如给出两端点的一阶导数值 $y_{0}^{\prime}, y_{N}^{\prime}$ 。

根据插值条件:

$$
S^{\prime}(a)=y_{0}^{\prime}, \quad S^{\prime}(b)=y_{N}^{\prime}
$$

以及有求左右偏导数得到新的方程:

$$
\begin{aligned}
& 2 M_{0}+M_{i}=\frac{6}{h_{i}}\left(\frac{y_{i}-y_{0}}{h_{i}}-y_{0}^{\prime}\right) \\
& M_{N-1}+2 M_{N}=\frac{6}{h_{N}}\left(-\frac{y_{N}-y_{N-1}}{h_{N}-y_{N}^{\prime}}\right)
\end{aligned}
$$


把式上述两式结合到式三次样条插值函数的基本方程组中，得到可解的线性代数方程组。

第三步, 求解方程组。按上述边界条件得到方程组如下式所示, 可用追赶法求解上式的三角对角方程 组, 用 Matlab 程序语言将下式转化为矩阵与矩阵相乘, 就能方便快捷地利用矩阵相除来求得系数 $M_{i}$, 再 求得 $S(x)$ 。

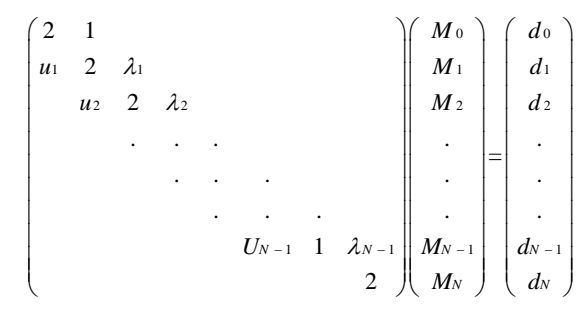

\section{2 曲线拟合误差分析}

由于数控插补的原因, 凸轮表面从微观上看应该呈多边形, 如图 1 所示。

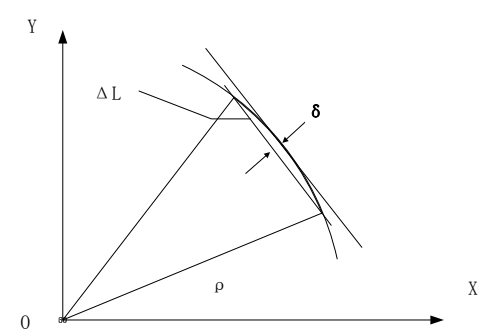

图 1 插补误差示意图

如果凸轮圆弧插补时的逼近误差为 $\delta$ 、插补周期为 $\mathrm{T}$ 、进给速度为 $\mathrm{F}$, 则可以推导出该曲线在逼近处的 误差 $\delta$ 与曲率半径 $\rho$ 的关系:

因为:

$$
\Delta L=\delta, \quad \mathrm{FT}=\rho \Phi
$$

所以:

$$
\delta=\rho-\sqrt{\rho^{2}-\left(\frac{\mathrm{FT}}{2}\right)^{2}} \approx \frac{(\mathrm{FT})^{2}}{8 \rho}=\frac{\rho(\Delta \phi)^{2}}{8}
$$

式中， $\Delta \phi$ 一升程表的角度间隔； $\rho$ 一凸轮轮廓半径。

本文选取的凸轮轮廓升程表角度间隔为 $1^{\circ}$, 在此基础上, 细化角度间隔为 $0.5^{\circ}$, 以获得更为光滑的轮 廓曲线, 运用三次样条函数插值法, 每隔 $0.5^{\circ}$ 插值后获得的凸轮轮廓节点数值, 把上述三次样条插值点导 入 Matlab 软件, 用绘图功能获得凸轮轮廓拟合曲线, 如图 2 所示

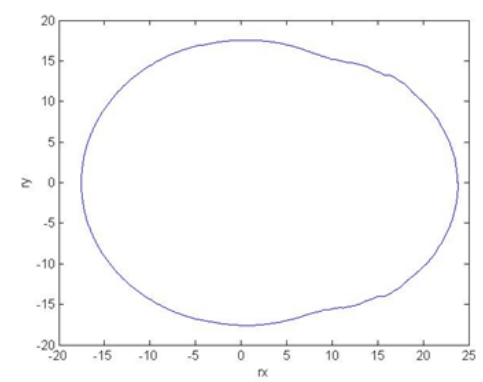

图 2 凸轮轮廓曲线的拟合 


\section{3 凸轮轮廓曲线的光顺处理}

光顺处理 ${ }^{[9]}$ 的阶段一般有两个, 先预光顺处理再精光顺处理。

\subsection{1 预光顺}

进行预光顺时，常用“差商替换微商”法，也就是采用各节点上升程的一阶差商及二阶差商 ${ }^{[10]}$ 来代替速 度 $d h / d \alpha$ 及加速度 $d h^{2} / d \alpha^{2}$, 具体公式如下:

$$
\begin{gathered}
\left.\frac{d h}{d \alpha}\right|_{\alpha=\alpha_{i}}=\frac{h\left(\alpha_{i+1}\right)-h\left(\alpha_{i-1}\right)}{\alpha_{i+1}-\alpha_{i-1}} \\
\left.\frac{d h^{2}}{d \alpha^{2}}\right|_{\alpha=\alpha_{i}}=\frac{h\left(\alpha_{i+1}\right)-2 h\left(\alpha_{i}\right)-h\left(\alpha_{i-1}\right)}{\left(\alpha_{i+1}-\alpha_{i-1}\right)^{2}}
\end{gathered}
$$

由于已知各节点处的升程值, 所以以上两式右端的值均可求出, 分别为 $h(\alpha)$ 的一阶差商和二阶差商。 这些差商的值随 $i$ 变化的情况, 基本上反映从动件速度和加速度的变化。

\subsection{2 精光顺}

预光顺过程中, 对二阶差商波动较大的区段所作的微小修正, 并由修正值重新计算差商值直至达到要 求的过程, 称为精光顺。

本文对凸轮轮廓精光顺的步骤如下:

(1) 根据节点 $\alpha_{0}, \alpha_{1}, \cdots, \alpha_{n}$ 和升程值 $h_{0}, h_{1}, \cdots, h_{n}$, 进行第一轮的三次样条插值, 得到差值函数 $\overline{h(\alpha)}$ 。

（2）应用各小区间的中点 $\left[\alpha_{\mathrm{i}}, \alpha_{\mathrm{i}+1}\right] / 2\left(i=0,1,2, \cdots, n\right.$-1) 再加上原来的两个端点 $\alpha_{0}$ 和 $\alpha_{n}$, 计 $n+2$ 个节点, 将它们由小到大排列, 并计作 $\beta_{0}, \beta_{1}, \cdots, \beta_{n+1}$ 和升程 $\overline{h_{0}}, \bar{h}_{1}, \cdots, \overline{h_{n+1}}$ 。

(3) 根据这些节点 $\beta_{1}, \beta_{2}, \cdots, \beta_{\mathrm{n}+1}$ 和升程 $\overline{\mathrm{h}_{0}}, \overline{\mathrm{h}_{1}}, \cdots, \overline{\mathrm{h}_{\mathrm{n}}}$, 利用前述方法重做三次样条插值, 得到新 的插值函数 $\overline{\overline{\mathrm{h}(\alpha)}}$ 。

(4) 计算 $\overline{\overline{\mathrm{h}(\alpha)}}$ 在原来节点 $\alpha_{0}, \alpha_{1}, \cdots, \alpha_{\mathrm{n}}$ 处的值 $\overline{\overline{\mathrm{h}\left(\alpha_{0}\right)}}, \overline{\overline{\mathrm{h}\left(\alpha_{1}\right)}}, \cdots, \overline{\overline{\mathrm{h}\left(\alpha_{1}\right)}}$, 并将其重记为 $\overline{\overline{\mathrm{h}_{0}}}, \overline{\overline{\mathrm{h}_{1}}}, \cdots, \overline{\overline{\mathrm{h}_{\mathrm{n}}}}$, 这就是原来所测升程值 $\mathrm{h}_{0}, \mathrm{~h}_{1}, \cdots, \mathrm{h}_{\mathrm{n}}$ 的首次修正值。

(5) 这样反复修正, 直到相邻两次修正值 $h_{i}$ 和 $\overline{\overline{h_{i}}}$ 的差满足条件:

$$
\left|h_{1}-\overline{\bar{h}}\right|<\xi \quad(i=1,2, \cdots, n-1)
$$

上式中, $\xi$ 是由要求预先确定的正数。

末次修正后的升程值就可作为光顺后的结果一凸轮从动件升程数据。由精光顺的结果即可构造出代替 $h(\alpha)$ 的三次样条插值函数 $\overline{\overline{h(\alpha)}}$ 。事实上, $h(\alpha)$ 在最后一次回弹中已经得出。然后, 再由 $h(\alpha)$ 的表达式就可 得到凸轮轮廓的曲线。

\section{4 光顺前后轮廓曲率曲线分析}

运用三次样条插值法拟合凸轮轮廓曲线时, 就可能出现对轮廓曲线影响很大的坏点。本文用三维软件 UG 对凸轮节点进行分析处理, 剔除坏点, 坏点剔除前后的样条曲率变化如图 3 和图 4 所示。 

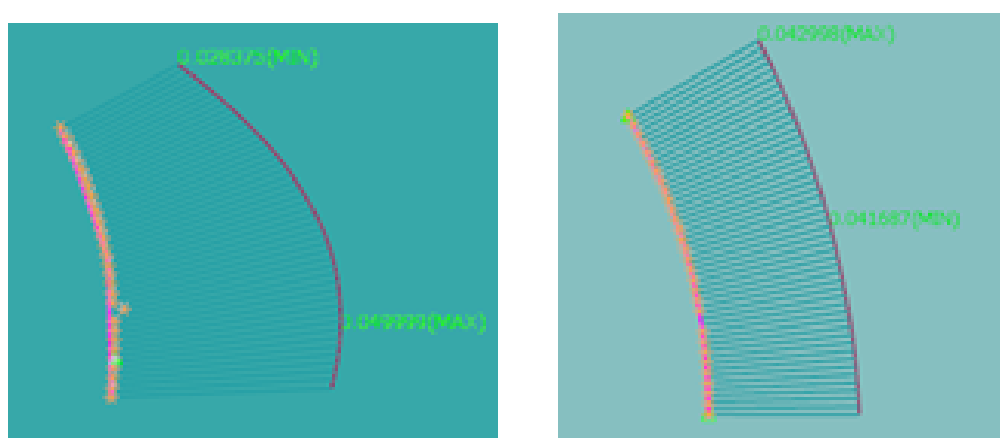

图 3 坏点剔除前的样条曲率变化 图 4 坏点剔除后的样条曲率变化

\section{2 结论}

本文阐述了凸轮轮廓曲线的插值拟合方法，运用三次样条插值方法来保证凸轮轮廓曲线的光滑和连 续。由于三次样条插值方法是通过插值点而不是逼近插值点, 更有利于提高凸轮轮廓精度, 所以本文通过 Matlab 软件采用三次样条插值方法对已知升程表数据进行插值计算。应用 UG 软件的曲率梳功能对凸轮的 轮廓曲线进行分析，通过对光顺前后曲率的比对分析，发现凸轮轮廓曲线得到了明显改善。

\section{3 致谢}

作者非常感谢国家自然科学基金资助项目(No. 51475142); 河南省教育厅自然科学研究项目（No. 12B520019) 给予的支持。

\section{参考文献:}

[1] 苌转, 王素梅, 海丰. 柴油机配气机构两种凸轮型线的设计及性能对比 [J]. 农业装备与车辆工程, 2012，50 (4)： $18-21$.

[2] 秦辉. N330 柴油机柴油机配气凸轮型线改进及优化设计 [D]. 武汉：武汉理工大学， 2011.

[3] 陈锟，刘克家，傅晓强等. 材料压缩变形过程应变场分布的函数表示法 [J]. 上海应用技术学院学报 (自然科学版), 2013, 13(2): 89-92.

[4] 黄浩洋，邓飞，隆振海等. 二维离散点集 Delaunay 三维网生长算法的改进 $[J]$. 电脑知识与技术，2016，12(23) : $188-191$.

[5] 魏逸飞，孙伟，黄涛等. 二次多项式拟合法和多面函数拟合法在 GNSS 高程测量中的应用 [J]。测绘与空间地理信 息, 2012, 35(12)：32-40.

[6] 赵蓝飞, 席志红. 基于三次样条插值的高动态范围成像方法 $[J]$. 计算机辅助设计与图形学报, $2015,27(7)$ : $1278-1289$.

[7] 陈敏，陶涛. 数控编程中列表曲线的拟合 [J]. 工艺与装备，2005，(6)：75-79.

[8] 姚仰新, 罗家洪, 庄楚强. 高等工程数学 [M]. 高等教育出版社, 2007 .

[9] 李耀辉，宣兆成，武志峰等. 二次代数曲面拼接中的光顺处理 [J]．计算机应用，2014，34(7)：2054-2057. $15-18$.

$[10]$ 王秀友, 范建中, 徐冬青等. 差商的性质及其应用探讨 $[J]$. 阜阳师范学院学报 (自然科学版)，2013，30(3)：

\section{Acknowledgements}

The authors gratefully acknowledge the National Nature Science Foundation (Project No. 51475142), Henan Provincial Department of Education Natural Science Research Project (Project No. 12B520019).

\section{References:}

[1] Chang Zhuan, Wang Sumei, Hai Feng. Design and Performance Contrast of the Two Cam Molded Lines of Diesel Engine Valve Mechanism [J]. Agricultural Equipment \& Vehicle Engineering. 2012, 50(4): 18-21.

[2] Qin Hui. Improvement and Optimal Design of Cam Molded Lines of N330 Diesel Engine Valve Mechanism [D]. Wuhan: Wuhan University of Technology. 2011.

[3] Chen Kun, Liu Kejia, Fu Xiaoqiang, et al. Function Representation of Strain Field Distribution 
in the Process of Material Compressive Deformation[J]. Journal of Shanghai Institute of Technology (Natural Science). 2013, 13(2): 89-92.

[4] Huang Haoyang, Deng Fei, Long Zhenhai, et al. Improvement of Delaunay Triangulation Network Growth Glgorithm for 2D Discrete Point Set [J]. Computer Knowledge and Technology. 2016, 12(23): 188-191.

[5] Wei Yifei, Sun Wei, Huang Tao, et al. Application of Quadratic Polynomial Fit and Polyhedral Function Fit Method to GNSS Height Measurement [J]. Geomatics \& Spatial Information Technology. 2012, 35(12): 32-40.

[6] Zhao Lanfei, Xi Zhihong. High Dynamic Range Imaging Method Based on Cubic Spline Interpolation [J]. Journal of Computer-Aided Design and Computer Graphics, 2015, 27(7): 1278-1289.

[7] Chen Min, Tao Tao. Tabular Curve Fitting in Numerical Control Programming [J].Technique \& Equipment, 2005, (6):75-79.

[8] Yao Yangxin, Luo Jiahong, Zhuang Chuqiang. Advanced Engineering Mathematics [M]. Higher Education Press, 2007.

[9] Li Yaohui, Xuan Zhaowu, Wu Zhifeng, et al. Fairing Processing in the Quadratic Algebra Curve Connection [J]. Journal of Computer Applications, 2014, 34(7): 2054-2057.

[10] Wang Xiuyou, Fan Jianzhong, Xu Dongqing, et al. Property of Difference Quotient and A Study of Its Application [J]. Journal of Fuyang Teachers College (Natural Science), 2013, 30(3): 15-18. 